\title{
Hydroxychloroquine-related skin discoloration
}

\author{
Jérôme Coulombe MD, Olivia Boccara MD
}

- Cite as: CMAJ 2017 February 6;189:E212. doi: 10.1503/cmaj.150622

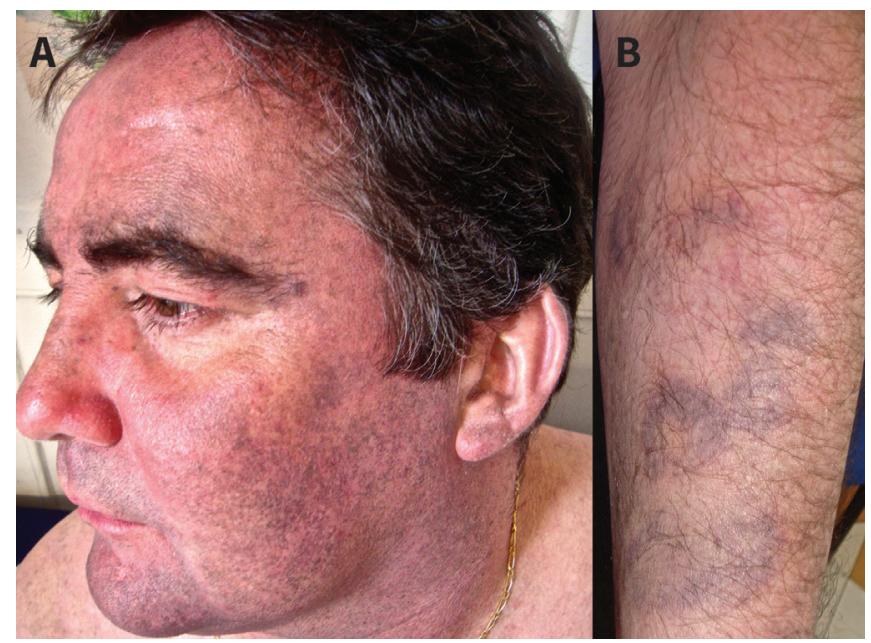

Figure 1: Hydroxychloroquine-related hyperpigmentation on the face $(A)$ and areas of previous bruising on the arm (B) of a 48-year-old man with systemic lupus erythematosus.

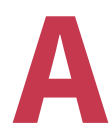

48-year-old man with a long-standing history of systemic lupus erythematosus presented with a three-month history of mottled blue-grey hyperpigmentation on his face and on areas of previous bruising on his arms and legs (Figure 1). There was no involvement of the mucous membrane or nails. His daily medication included hydroxychloroquine and prednisone in addition to mycophenolate mofetil, warfarin, clopidogrel, lercanidipine, ramipril and amlodipine. He had been taking hydroxychloroquine for more than 20 years. There was no other drug exposure. His serum iron load and amyloid levels were normal, as were findings on a recent ophthalmologic examination. Skin biopsy from the upper arm showed superficial dermal, yellow-brown, nonrefractile and coarsely granular pigment deposition interstitially and within histiocytes. Results of Fontana-Masson staining for melanin and Perls Prussian blue staining for iron were positive.

The incidence of hydroxychloroquine-related hyperpigmentation in patients with systemic lupus erythematosus is about $7 \%$, but unlike hydroxychloroquine-related retinopathy, there is no clear association with cumulative dose or duration of use. ${ }^{1}$ Roughly half of cases occur in the first five years of treatment. Macular and patchy blue-grey to black discoloration has been described on the gums, palate, nails, face and arms, and invariably on the shins. ${ }^{2}$

The mechanism for hyperpigmentation is poorly understood. Risk factors appear to be linked to bruising, corticosteroid use, oral anticoagulants, antiplatelet agents, antiphospholipid syndrome and skin trauma. ${ }^{1}$ Histology usually shows superficial dermal deposition of brown-pigmented granules of iron or melanin, or both.

The risk of hydroxychloroquine-related retinopathy associated with skin hyperpigmentation is unknown. ${ }^{3}$ The risk of eye toxicity increases sharply toward $1 \%$ after five to seven years of hydroxychloroquine use, or a cumulative dose of $1000 \mathrm{~g} .{ }^{4}$

Improvement of the hyperpigmentation a few months after hydroxychloroquine is stopped has been reported, but complete clearance is rare. ${ }^{1}$ Cautious use of the Q-switched ruby laser has shown promise in the treatment of nonmelanotic hyperpigmentation in limited case reports. ${ }^{5}$ Our patient's skin discoloration partially resolved four months after switching to chloroquine.

\section{References}

1. Jallouli M, Francès $C$, Piette JC, et al. Hydroxychloroquine-induced pigmentation in patients with systemic lupus erythematosus: a case-control study. JAMA Dermatol 2013;149:935-40.

2. Dereure O. Drug induced skin pigmentation: epidemiology, diagnosis and treatment. Am J Clin Dermatol 2001;2:253-62.

3. Tuffanelli D, Abraham RK, Dubois El. Pigmentation from antimalarial therapy: its possible relationship to the ocular lesions. Arch Dermatol 1963;88:419-26.

4. Marmor MF, Kellner U, Lai TY, et al.; American Academy of Ophthalmology. Revised recommendations on screening for chloroquine and hydroxychloroquine retinopathy. Ophthalmology 2011;118:415-22.

5. Becker-Wegerich PM, Kuhn A, Malek L, et al. Treatment of nonmelanotic hyperpigmentation with the Q-switched ruby laser. J Am Acad Dermatol 2000;43:272-4.

\section{Competing interests: None declared.}

This article has been peer reviewed.

The authors have obtained patient consent.

Affiliations: Department of Dermatology (Coulombe), Centre hospitalier universitaire Sainte-Justine, Montréal, Que.; Department of Dermatology (Boccara), Hôpital Necker - Enfants malades, Paris, France

Correspondence to: Jérôme Coulombe, jerome.coulombe@ umontreal.ca

Clinical images are chosen because they are particularly intriguing, classic or dramatic. Submissions of clear, appropriately labelled highresolution images must be accompanied by a figure caption. A brief explanation ( 300 words maximum) of the educational significance of the images with minimal references is required. The patient's written consent for publication must be obtained before submission. 